Cultures \& Conflits

03 | automne 1991

Mafia, drogue et politique

\title{
Editorial - L'interpénétration des jeux mafieux et politiques
}

Didier Bigo et Michel Dobry

\section{OpenEdition}

1 Journals

\section{Édition électronique}

URL : http://journals.openedition.org/conflits/2014

DOI : $10.4000 /$ conflits. 2014

ISSN : $1777-5345$

Éditeur :

CCLS - Centre d'études sur les conflits lilberté et sécurité, L'Harmattan

Édition imprimée

Date de publication : 17 octobre 1991

ISSN : 1157-996X

Référence électronique

Didier Bigo et Michel Dobry, «Editorial - L'interpénétration des jeux mafieux et politiques », Cultures \& Conflits [En ligne], 03 | automne 1991, mis en ligne le 31 mars 2006, consulté le 30 mars 2021. URL:

http://journals.openedition.org/conflits/2014 ; DOI : https://doi.org/10.4000/conflits.2014

Ce document a été généré automatiquement le 30 mars 2021.

Creative Commons License 


\title{
Editorial - L'interpénétration des jeux mafieux et politiques
}

\author{
Didier Bigo et Michel Dobry
}

1 Témoignages de victimes, révélations spectaculaires sur l'implication de hautes personnalités, stigmatisation de certains pays producteurs, campagnes de publicité pour les programmes de lutte anti-drogue se succèdent à un rythme accéléré. Pourtant, au-delà de cette agitation et de ces révélations ponctuelles, qui posent immédiatement la question des liens avec le politique, peu de travaux universitaires ont abordé le sujet.

2 Crainte d'un "objet sale", d'une contamination de la suspicion qui, de l'objet drogue remonterait vers le chercheur ? Absence de sources fables qui condamneraient l'universitaire à se muer en journaliste d'investigation ou en auxiliaire de police ? Si médecins, criminologues et, récemment, économistes ont apporté leurs contributions à l'étude du phénomène de la toxicomanie et de ses implications, les spécialistes de sociologie et de science politique étaient restés quasiment muets jusqu'à présent.

3 Ce numéro 3 de Cultures et Conflits a voulu montrer qu'une démarche de science politique avait sa raison d'être sur ce terrain car les questions posées par les rapports "mafia, drogue et politique" touchent au fondement même de la science politique. A commencer bien sûr par la question de la monopolisation par l'État de la violence organisée légitime.

4 L'observation de ce terrain bouscule, à cet égard, aussi bien certitudes que définitions, fussent-elles idéal-typiques. Les luttes concurrentielles pour le contrôle des populations dans les zones de production des plantes à drogue (coca, opium, kif) entre les organisations de type mafieux et le gouvernement central, parfois compliquées par l'existence de groupes de guérilla et par l'autonomisation des forces répressives, obligent en effet à revenir sur un certain nombre de postulats. Une étude attentive des marges tolérées par l'État dans l'usage d'une violence qui prend une signification politique montre que les transactions, voire les collusions, l'emportent souvent sur la prétention à monopoliser l'usage de la force. Que ce soit au Pakistan, en Birmanie, aux Philippines, en Malaisie, au Pérou ou en Colombie, le gouvernement central est souvent bien en peine de se faire obéir des institutions répressives qui agissent, sans doute 
encore plus qu'ailleurs, en fonction de leurs intérêts propres. De plus, il existe certains cas où, non seulement le gouvernement a du mal à contrôler l'ensemble du territoire, mais où il ne le désire pas car cela lui permet de profiter des espaces d'illégalité (drogue, armes, contrebandes diverses) ainsi créés à ses frontières.

5 En outre, l'analyse fait également ressortir un phénomène qui n'appartient pas qu'au passé lointain de nos sociétés, celui de l'émergence de "zones désétatisées", véritables trous, brèches dans le maillage interétatique planétaire, remettant en cause notre vision des relations internationales. Aussi bien dans le Liban des années 80, la zone du Triangle d'or aux frontières de la Birmanie de la Thaïlande et du Laos, le Croissant d'or entre Pakistan et Afghanistan, la "zone amazonienne cocalera" entre le Pérou, la Bolivie, le Brésil et la Colombie que dans le Rif marocain et depuis peu dans certains territoires de l'Afrique subsaharienne abandonnés par des dirigeants sans moyens, on voit des acteurs non étatiques (groupes de guérilla, mafias, narco-trafiquants...) posséder les attributs, sinon de l'État, du moins d'un pouvoir politique fortement centralisé qui sait se faire obéir des populations, de façon plus ou moins coercitive. Ainsi, même non reconnues internationalement, ces zones existent depuis des années, et des groupes politiques les contrôlent en se substituant à l'État soit par la force, soit par entente tacite, soit encore grâce à des alliances avec les dirigeants des États voisins.

6 L'usage de la coercition sur les populations n'y est d'ailleurs pas forcément plus intense qu'ailleurs : la drogue étant un produit fortement lucratif, la redistribution aux producteurs, même extrêmement limitée, peut parfois suffire pour améliorer nettement leurs conditions de vie et les convaincre de continuer ce type de production sous la houlette de groupes de trafiquants ou/et de groupes de guérilla ou/et de groupes paramilitaires qui contrôlent les territoires en question. Seulement, la monopolisation de l'usage de la violence est assez fragile dans ces zones. Elle est remise en cause à la première occasion par les concurrences entre ces groupes d'acteurs "privés" qui se spécialisent dans l'offre de sécurité et la détention des moyens de la violence organisée. Mais, même fragiles, ces entreprises de monopolisation de la violence semblent parfois bénéficier de l'adhésion des dominés. Ceci pose le problème réel des capacités des acteurs à se légitimer (si tant est que cela soit toujours nécessaire) et à mobiliser les populations des zones de production. Comment se déploient ces discours de légitimation et comment s'articulent-ils sur les mythes, les valeurs, les pratiques sociales des populations ? C'est le premier aspect des processus de légitimation. La culture de la coca correspond certes à un type de culture spéculative qui se rattache pour les populations sud-américaines aux grandes spéculations du siècle dernier (hévéas, café...). Il existe de plus une mémoire historique de ce type de production et le producteur bolivien ou péruvien n'éprouve pas forcément un sentiment de culpabilité. Il en va de même pour les populations qui cultivent du pavot ou du kif dans le Triangle d'or ou le Croissant d'or. Mais, comment les populations vivent-elles le fait de cultiver ces plantes et de les transformer en pâte base de cocaïne ou en héroïne ? Sous le mode de la bonne conscience à travers la "tradition", sous celui de la culpabilité morale, sous celui des impératifs de survie économique, de l'abandon de l'État central et de sa répression incontrôlée... ? Nous nous proposons de revenir sur cette question dans un prochain numéro.

7 L'autre aspect des processus de légitimation, souvent négligé lorsqu'on se focalise sur les discours, les valeurs, les cultures, c'est ce qui se joue dans les échanges, voire dans l'interpénétration entre les organisations de type mafieux, les groupes dirigeants et les 
professionnels de la politique. Dans certains pays, telle la Colombie, la vision médiatique qui nous est proposée est celle de deux groupes homogènes, séparés, en lutte l'un contre l'autre. Le rapport de force aurait fait triompher de justesse l'État contre le cartel de Medellín. Seulement des études plus serrées montrent à quel point, même dans ce pays où le président a voulu agir, les liens entre les groupes dirigeants (gouvernement, parlementaires, "oligarchie" locale...) et le cartel de Medellín ainsi que celui de Cali sont étroits. Ailleurs, en Thaïlande, aux Philippines, au Pakistan, au Liban... les trafics ne pourraient avoir l'ampleur qu'ils ont si les institutions policières ou militaires n'étaient pas compromises dans le trafic, au point parfois d'en être les véritables patrons. Les dirigeants gouvernementaux peuvent aussi, loin des discours de dénonciation contre le fléau de la drogue, être les principaux organisateurs du troc, surtout dans les pays de transit. Certains pays du Golfe ou de la région du Croissant d'or, des pays d'Afrique subsaharienne, des pays du Triangle d'or, des pays du ProcheOrient, sans oublier certains pays occidentaux, via le blanchiment de l'argent, peuvent connaître ce phénomène de "réseau" qui traverse la société entière et relie les groupes criminels aux plus hautes instances politiques. Le Panama de Noriega en a été l'exemple caricatural. Mais il en va de même en Italie par exemple avec la Camorra qui investit les postes politiques locaux. Il faut donc être prudent avec le vocabulaire. Le jeu des dénominations, des labelisations paralyse souvent les analyses criminologiques ou gouvernementales en faisant croire à des groupes bien distincts là où l'analyse sociologique montre la dynamique en réseau des acteurs mafieux et politiques. Il faut donc s'interroger sur les formes prises par les diverses organisations de type mafieux en voyant leur degré de fermeture (rapport à la territorialité, aux structures sociales, aux formes de solidarité...), leurs capacités d'adaptation (dimension historique de l'urbanisation, extension des marchés, internationalisation...), les répertoires d'actions qu'elles utilisent (menace et usage de violence, participation aux marchés illégaux et légaux, gestion concurrentielle de la protection, modalités d'extorsion...), les transactions qu'elles opèrent avec les autres pouvoirs... et les restructurations sociétales qu'elles impliquent tant sur le plan interne qu'international.

Ces dynamiques en réseaux qui, justement, avec l'acheminement et la commercialisation de la drogue, perturbent les logiques territoriales, obligent les mafias qui se spécialisent dans ces trafics à abandonner certaines de leurs bases sociales, à s'ouvrir, à se délocaliser et à dépasser les cadres nationaux. Elles s'inscrivent alors dans des courants d'échanges transnationaux où les jeux d'alliance et de conflit que nous avons vu à l'échelle locale prennent une dimension internationale, tellement cruciale pour certains pays et certains dirigeants, qu'il y va de leur avenir économique et politique. Il est difficile de définir la "nébuleuse transnationale", le réseau mafieux qui traverse les frontières de l'illégal et du léga1 aussi facilement que les frontières terrestres, aériennes ou maritimes. Son étude en est délicate. Si l'on connaît un peu les organisations de type mafieux d'un certain nombre de pays, quels sont leurs contacts ? Comment gèrent-elles le rapport entre assise territoriale qui leur assure un minimum de base sociale et transnationalité des réseaux de la drogue qui les insère dans une véritable économie politique mondiale. $\mathrm{Y}$ a-t-il par exemple un "nationalisme" des groupes narco-colombiens? Peuvent-ils, même riches, accepter de partir de leur pays ? Ne préfèrent-ils pas pour eux la prison mais pour leurs enfants une réussite sociale dans "leur" pays ? N'est-ce pas aussi ce qui pourrait expliquer le retrait relatif des organisations mafieuses siciliennes et napolitaines dans le trafic de drogue ? Celui-ci n'est-il pas de plus en plus pris en charge par divers segments des classes politiques au 
point que le terme de criminalisation du politique ne serait plus simplement métaphorique ? L'implication de nombreuses personnes au sein des groupes dirigeants sur la planète laisse peu d'espoir aux plans classiques de lutte contre la drogue.

9 Finalement, la drogue, comme les autres sources de revenus occultes, devient une ressource logique dans l'enrichissement nécessaire au clientélisme politique et donc dans les jeux politiques, qu'ils soient violents ou non (coups d État, élections). Plus généralement, la captation de ressources extérieures, les possibilités d'enrichissement facile, l'insertion dans des réseaux hautement rémunérateurs, peuvent être perçus comme un moyen de rester dans le jeu politique et le commerce international, comme un moyen d'éviter la marginalisation et donc de retrouver certaines formes de légitimité aux yeux des acteurs subordonnés. Ainsi, la drogue n'est-elle, aux yeux de ce(s) réseau(x) mafieux/politique u'un moyen parmi d'autres (affaires, trafic d'armes...) de s'enrichir ou/et de rester au pouvoir.

Reste à préciser l'ampleur de ce(s) réseau(x) qui n'épargne(nt) pas non plus les pays occidentaux.

\section{INDEX}

Mots-clés : mafias et crime organisé, sciences politiques, drogue, politique 